\title{
Minimal area submanifolds in AdS $\times$ compact
}

\section{Robin Graham ${ }^{a}$ and Andreas Karch ${ }^{b}$}

\author{
${ }^{a}$ Department of Mathematics, University of Washington, \\ Seattle, WA 98195-4350, U.S.A. \\ ${ }^{b}$ Department of Physics, University of Washington, \\ Seattle, WA 98195-1560, U.S.A. \\ E-mail: robin@math.washington.edu, karch@phys.washington.edu
}

\begin{abstract}
We describe the asymptotic behavior of minimal area submanifolds in product spacetimes of an asymptotically hyperbolic space times a compact internal manifold. In particular, we find that unlike the case of a minimal area submanifold just in an asymptotically hyperbolic space, the internal part of the boundary submanifold is constrained to be itself a minimal area submanifold. For applications to holography, this tells us what are the allowed "flavor branes" that can be added to a holographic field theory. We also give a compact geometric expression for the spectrum of operator dimensions associated with the slipping modes of the submanifold in the internal space. We illustrate our results with several examples, including some that haven't appeared in the literature before.
\end{abstract}

Keywords: D-branes, AdS-CFT Correspondence, Differential and Algebraic Geometry

ARXIV EPRINT: 1401.7692 


\section{Contents}

1 Introduction 1

2 Product submanifolds and their deformations 5

2.1 Product submanifolds 5

2.2 Jacobi operator 5

$\begin{array}{lll}2.3 & \text { Fluctuation in the internal space } & 7\end{array}$

2.4 Fluctuation in AdS 8

3 Minimality constraint on internal submanifold $\quad 10$

4 Examples $\quad 14$

$\begin{array}{ll}4.1 & \text { Slipping modes on spheres } \\ \end{array}$

$\begin{array}{ll}\text { 4.1.1 The flavor D7 brane } & 14\end{array}$

$\begin{array}{ll}\text { 4.1.2 General AdS times sphere example } & 15\end{array}$

$\begin{array}{lll}4.2 & \text { Disjoint boundaries } & 17\end{array}$

$\begin{array}{lll}\text { 4.2.1 Wilson lines with internal motion } & 17\end{array}$

$\begin{array}{lll}4.2 .2 & \text { Rotating spheres } & 18\end{array}$

$\begin{array}{lll}\text { 4.2.3 Interaction between internal and hyperbolic factors } & 19\end{array}$

\section{Introduction}

The gauge/gravity correspondence [1-3] or "holography" for short, provides a large class of solvable models of strong coupling dynamics. These solvable toy models are being employed to understand qualitative aspects of an ever growing array of physics questions, spanning from nuclear to atomic and condensed matter physics. The mathematics underlying holographic techniques is 28 years old and predates its application to physics [4]. Holography relates strongly coupled quantum field theories in $n$ spacetime dimensions to a gravitational problem in one higher dimension. The solution of the system via holography therefore becomes essentially a geometric problem: the geometry of the extra dimension encodes all the properties of the strongly coupled physical system. In the simplest cases, the higher dimensional space needs to be of a very special geometric type: a product manifold with one factor that is an asymptotically hyperbolic Einstein space and the other factor a compact "internal" space. In essence, the problem is one of constructing solutions to a non-linear second order partial differential equation (encoding the Einstein condition) subject to certain asymptotic boundary conditions. The asymptotic behavior of such spaces at infinity has been described in in [4-6]. 
There are several questions within these holographic toy models for which one, in addition to the background geometry, is also interested in finding a minimal ${ }^{1}$ area submanifold within that space. Let us briefly recall three classes of questions that rely on minimal area submanifolds.

The first appearance of minimal area submanifolds in holography was in the context of Wilson lines [7, 8]. A Wilson line measures the response of the strongly coupled system to inclusion of an external test particle, following a predescribed worldline. To calculate the expectation value of the operator describing this insertion, the holographic recipe is to calculate the area of the minimal area surface in the bulk ending on the worldline.

A second example of an application of minimal area submanifolds in holography is probe flavor branes [9]. Flavor branes are needed to incorporate quarks into holographic models of QCD; for condensed matter applications they can introduce the charge carriers (the electrons) into a strongly coupled phonon bath modeled, for example, by $\mathcal{N}=4$ SuperYang Mills (SYM). The extra degrees of freedom added to the quantum field theory can either live in the whole $n$ dimensional field theory spacetime, or only on a $k$ dimensional subspace (a "defect"). The holographic description requires a minimal area submanifold ending on the location of the defect. In addition, different flavor branes wrap different submanifolds of the internal space, corresponding to different matter content and interactions of the extra degrees of freedom added to the field theory. The worldvolume of the flavor brane only has to be a minimal area submanifold when regarded as a submanifold of the full product spacetime that constitutes the holographic dual, not separately as a submanifold of the hyperbolic and the compact factor. The interplay of the shape of the submanifold in the internal and hyperbolic factor are crucial to model even the simplest physical parameters such a theory should have, for example the mass of the extra fields. The Wilson line can be viewed as a special case of a flavor brane, where the matter added is a $0+1$ dimensional defect, with the degree of freedom living on it being the external test quark.

The reason that in these cases the original geometric question of finding an Einstein manifold gets replaced by a minimal area problem is the "probe approximation" inherent in this construction. In general, changing the theory by adding extra matter would require to re-solve the system of coupled differential equations describing the bulk geometry. The tension of the flavor brane gives rise to a non-zero stress tensor that appears as a source on the right-hand side of the bulk Einstein equations. The worldvolume of the brane however wants to minimize its area in this backreacted geometry. This way one finds a new background for the new field theory. In the limit that the extra degrees of freedom added are much fewer than the degrees of freedom in the original theory, ${ }^{2}$ the stress tensor associated to the extra matter is negligible and consequently it does not backreact on

\footnotetext{
${ }^{1}$ Following the tradition in the mathematical literature, by "minimal" area submanifold we simply mean a submanifold whose area doesn't change at linear order under small fluctuations. They can be minima, maxima or saddles of the area functional. A minimal submanifold is referred to as stable if the second order variation of area is positive definite. In particular, stable minimal submanifolds are local minima of the area functional.

${ }^{2}$ In cases where the strongly coupled field theory is a non-Abelian gauge theory with $\mathrm{SU}(N)$ gauge group, this is typically the case when the added matter is in the fundamental representation of the gauge group. While there are of the order $N^{2}$ gauge fields, there are only of order $N$ matter fields.
} 
the geometry. The flavor brane simply minimizes its own worldvolume area in a fixed background geometry.

The third and most recent example of studying minimal area problems in holography is entanglement entropies. While we will keep our discussion in the Euclidean setting unless explicitly mentioned otherwise, entanglement entropy is intrinsically tied to Lorentzian signature. If at a given time $t_{0}$ the $n-1$ dimensional field theory space is separated into two regions by a $n-2$ dimensional surface, one can, at that instant, associate an entropy to the field theory living in one of the regions by tracing over the degrees of freedom in the other region. This is called the entanglement entropy. The holographic proposal of [10] demands that the corresponding entanglement entropy is given by the area of a minimal area submanifold ending on the surface dividing the two regions, measured in Planck units (that is $S=A /(4 G)$, where $S$ is the entanglement entropy, $A$ the area and $G$ Newton's constant in the bulk). For the special case that the bulk is static, for example if it is $n+1$ dimensional Anti de-Sitter space $\left(\mathrm{AdS}_{n+1}\right)$, the minimal area in the bulk will also be at the same fixed time $t=t_{0}$; the problem then reduces to finding a minimal area submanifold in the $t=t_{0}$ submanifold of $\mathrm{AdS}_{n+1}$, which itself is an $n$ dimensional hyperbolic space. Note that in this proposal the bulk minimal area should be co-dimension 2, just as the field theory surface it ends on. Consequently, in the case where the bulk is a product manifold this minimal area submanifold always wraps the entire internal space. Unlike the case of flavor branes, here the problem essentially reduces to that of finding minimal area submanifolds in the asymptotically hyperbolic Einstein space itself.

The goal of our work is to fully describe the asymptotic structure of the most general minimal area submanifold in asymptotically hyperbolic Einstein spaces times a compact internal manifold which itself is asymptotic to a product of a submanifold in each factor. The corresponding problem in an asymptotically hyperbolic Einstein space itself has been solved in [11]. In particular for the case of flavor branes, it is crucial to address the question to what extent this picture changes when one asks for minimal areas in the product spacetime. The resulting structure is indeed much richer than what one gets simply in an asymptotically hyperbolic Einstein space.

This paper is mostly dedicated to a physics audience. We derive two main results. We find an interesting new constraint and we give an explicit geometric formula for the operator dimensions of operators dual to deformations of the cycle. In a more mathematical companion paper we will analyze the general formal asymptotics of minimal area submanifolds asymptotic to products.

Our constraint restricts the asymptotic form the submanifold can have. While the submanifold occupied by a defect flavor brane in the field theory dimensions is arbitrary, the asymptotic submanifold it wraps in the internal space has to be minimal itself. This constrains the form of potential flavor branes one may wish to add to the geometry. For the operator spectrum, we consider submanifolds that can be viewed as a first order deformation of a product submanifold, with the requirement that the deformation vanishes asymptotically. According to the AdS/CFT dictionary, these dimensions correspond in the bulk to the indicial roots for the linearized minimal submanifold equations. We show that the dimensions are governed by two geometric operators defined by the geometry of 
the minimal submanifold in the internal space: the scalar Laplace operator and the Jacobi operator. The derivations of both the minimality constraint and the operator spectrum only require the spacetime background metric to be asymptotically hyperbolic; they do not use the Einstein condition. We also work out several novel examples of minimal area submanifolds; in particular we give examples of submanifolds where perturbations of the product submanifold are turned on in both factors simultaneously.

The organization of this paper is as follows: in the next section we give the description of the full asymptotic data starting from the case of a product submanifold and working out its deformations. In section 3 we derive the constraint that the boundary internal submanifold must be minimal. In section 4 we give several examples as well as their physical interpretation.

An important aspect of the minimal area problem is the calculation of the renormalized area. Due to the singularity of the metric near the boundary of asymptotically hyperbolic spaces, the area of all the submanifolds we consider is divergent. To assign a finite answer for the actual area one has to carefully understand the various divergent contributions to the area and cancel them with appropriate local counterterms. In the physics literature this is known as "holo-RG". The procedure is well-understood for minimal area submanifolds of asymptotically hyperbolic Einstein spaces. When internal variables are allowed, the program has been carried out for special examples in [12]. We leave for the future the study of the renormalized area for the general minimal area problem in spaces with internal variables.

Notation. We consider background spacetimes of the form $X^{n+1} \times K^{m}$, where $X^{n+1}$ is a $n+1$ dimensional manifold with asymptotically hyperbolic metric $g_{X}$ and boundary $\partial X=M$ and $K^{m}$ is a compact $m$ dimensional manifold with metric $g_{K}$. We take the metric on $X^{n+1} \times K^{m}$ to be the product

$$
g=g_{X}+g_{K}
$$

and we work in Euclidean signature. The assumption that $g_{X}$ is asymptotically hyperbolic means that in an appropriate choice of coordinates it can be written in the form

$$
g_{X}=\frac{d r^{2}+\bar{g}_{r}}{r^{2}}
$$

where $\partial X=\{r=0\}$ and $\bar{g}_{r}$ is a 1-parameter family of metrics on $M$ smooth in $r$ up to $r=0$.

Let us choose submanifolds $N^{k} \subset M$ and $\Sigma^{l} \subset K$. We are interested in minimal submanifolds $Z \subset X \times K$ with $\partial Z=N \times \Sigma \subset M \times K$. For simplicity, we take all our submanifolds to be embedded (no self-intersections) and regular at infinity. We can choose local coordinates $\left(x^{\alpha}, u^{\alpha^{\prime}}\right)$ on $M$ and $\left(s^{A}, t^{A^{\prime}}\right)$ on $K$ in such a way that $N \subset M$ is given by $N=\left\{u^{\alpha^{\prime}}=0\right\}$ and $\Sigma \subset K$ is given by $\Sigma=\left\{t^{A^{\prime}}=0\right\}$, and further

$$
\left.\bar{g}_{\alpha \beta^{\prime}}\right|_{r=0, u=0}=\left.g_{A B^{\prime}}\right|_{t=0}=0 \text {. }
$$

That is, the $t$ and $u$ coordinates on the boundary are "orthogonal" to the defect. In such coordinates in which (1.1) also holds, our minimal submanifold $Z$ is described by giving the $u$ 's and $t$ 's as functions of $(x, r, s)$, and the boundary condition reads $u=0, t=0$ at $r=0$. 


\section{Product submanifolds and their deformations}

\section{$2.1 \quad$ Product submanifolds}

The simplest examples of minimal area submanifolds in asymptotically AdS times compact product spaces are submanifolds where not just the boundary of $Z$ is a product manifold $\partial Z=N \times \Sigma$, but where $Z$ itself is a product $Z_{0}=N^{\prime} \times \Sigma$ with $\partial N^{\prime}=N$. In fact, any product of the form $N^{\prime} \times \Sigma$ is a minimal area submanifold in $X \times K$ as long as $N^{\prime}$ is a minimal area submanifold in $X$ and $\Sigma$ a minimal area submanifold in $K$. The converse is true too: if $N^{\prime} \times \Sigma$ is minimal in $X \times K$, then $N^{\prime}$ must be minimal in $X$ and $\Sigma$ minimal in $K$.

A general analysis of the formal asymptotics of minimal submanifolds $N^{\prime}$ of $X$ has been performed already in [11]. In this case, the local data that needs to be specified near the boundary is an arbitrary submanifold $\partial N^{\prime}=N$ of the boundary manifold $M$. The shape of $N$ is completely unconstrained. A minimal submanifold $N^{\prime}$ necessarily intersects $\partial X$ orthogonally. One describes $N^{\prime}$ by giving $u^{\alpha^{\prime}}$ as a function of $x$ and $r . u^{\alpha^{\prime}}(x, r)$ is then specified as a power series in $r$ (and its logarithm). The coefficient of $r^{k+2}$ is locally undetermined. It is typically fixed by some global requirements, such as smoothness, on the submanifold. Also there can be different minimal submanifolds $N^{\prime}$ with the same boundary submanifold $N$ and these will typically have different values for the $r^{k+2}$ coefficient. Once the coefficient of $r^{k+2}$ is fixed, there is no more freedom in the series expansion defining $u(x, r)$. In stark contrast, the internal factor $\Sigma$ already needs to be minimal in $K$ to begin with. So for the case of product submanifolds, we say that the boundary data for the minimal product submanifold $Z_{0}$ consists of an arbitrary submanifold $N$ of $M$ together with a minimal submanifold of $K$.

In the following we want to analyze the generic structure of minimal submanifolds $Z$ that are obtained by infinitesimal perturbations of a product minimal submanifold $Z_{0}=$ $N^{\prime} \times \Sigma$. The requirement that our submanifold has as its boundary a product $N \times \Sigma$ means that we require that the perturbation goes to zero at the boundary; it need not be small in the bulk of the space and need not itself be of product form. In the remainder of this section we will identify the complete local data that determines such an infinitesimally perturbed product submanifold. Elsewhere we will show that relaxing the requirement that the submanifold can be written as the deformation of a product does not lead to any additional freedom.

\section{$2.2 \quad$ Jacobi operator}

The spectrum of small fluctuations around a minimal submanifold is governed by the Jacobi operator $\mathcal{J}$ of the submanifold. We digress briefly to review the Jacobi operator since it plays a central role in our discussion. A more detailed discussion can be found, for instance, in [13].

In general, suppose that $Z$ is a submanifold of a space $X$ with a Riemannian metric $g_{X}$. Perturbations of $Z$ can be described by 1-parameter families of maps $F_{t}: Z \rightarrow Z_{t} \subset X$ for small $t$ satisfying $F_{0}=$ Identity. The derivative $\delta Z=\left.\partial_{t} F_{t}\right|_{t=0}$ can be interpreted as a vector field defined on $Z$, which we assume is everywhere normal to $Z$ since tangential vector fields correspond to reparametrizations of $Z$. The condition that $Z$ is minimal is 
the requirement that $\left.\partial_{t} A\left(Z_{t}\right)\right|_{t=0}=0$ for all maps $F_{t}$, where $A$ denotes area. This can be expressed by the vanishing of the mean curvature vector of $Z$. Recall that the mean curvature vector is defined as follows. The second fundamental form, or extrinsic curvature, $\mathcal{F}$ of a submanifold $Z \subset X$ is the symmetric quadratic form on $T Z$ with values in the normal bundle $\mathcal{N}$ given by $\mathcal{F}(V, W)=\left({ }^{X} \nabla_{V} W\right)^{\perp}$. Here ${ }^{X} \nabla$ denotes the Levi-Civita connection of $g_{X}$ and ${ }^{\perp}$ the component normal to $Z$. The mean curvature vector $H$ of $Z$ is the trace of the second fundamental form with respect to the induced metric on $Z: H=\operatorname{tr}_{g_{Z}} \mathcal{F}$. So $H$ is a section of $\mathcal{N}$ on $Z$. Then

$$
\left.\partial_{t} A\left(Z_{t}\right)\right|_{t=0}=-\int_{Z}\langle H, \delta Z\rangle d v_{g_{Z}}
$$

so $Z$ is minimal is the same as $H=0$.

Suppose now that $Z$ is minimal. The second derivative of the area function can be expressed as

$$
\left.\partial_{t}^{2} A\left(Z_{t}\right)\right|_{t=0}=\int_{Z}\langle\mathcal{J} \delta Z, \delta Z\rangle d v_{g_{Z}} .
$$

Here $\mathcal{J}$ is the Jacobi operator of $Z$, a differential operator acting on sections of $\mathcal{N}$. The Jacobi operator can be expressed in invariant terms as follows:

$$
\mathcal{J}=\nabla^{*} \nabla-\mathcal{R}-\mathcal{F}^{2}
$$

We explain each of the three summands. The first, $\nabla^{*} \nabla$, is the normal bundle Laplacian. The Levi-Civita connection ${ }^{X} \nabla$ of $g_{X}$ induces a connection $\nabla$ on $\mathcal{N}$ defined by $\nabla_{V} U=$ $\left({ }^{X} \nabla_{V} U\right)^{\perp}$. Viewing $\nabla: \Gamma(\mathcal{N}) \rightarrow \Gamma\left(\mathcal{N} \otimes T^{*} Z\right), \nabla^{*}$ denotes the adjoint operator and $\nabla^{*} \nabla$ their composition. Alternately, $\nabla^{*} \nabla$ may be expressed as

$$
\left(\nabla^{*} \nabla U\right)^{a^{\prime}}=-g^{a b} \nabla_{a} \nabla_{b} U^{a^{\prime}} .
$$

Unprimed indices correspond to tangent directions to $Z$ and primed indices to normal directions. On the right-hand side, $\nabla_{a}$ denotes the normal bundle connection coupled with the connection on $T Z$ induced by the Levi-Civita connection on $X$. The second term $\mathcal{R}$ in (2.1) is a zeroth order term; it is the linear transformation of the normal space at each point given by

$$
(\mathcal{R} U)^{a^{\prime}}=R^{a a^{\prime}}{ }_{a b^{\prime}} U^{b^{\prime}},
$$

where $R$ denotes the curvature tensor of the background metric $g_{X}$. $\mathcal{R}$ is perhaps best viewed as a partial mixed version of the Ricci tensor: it is the normal part of the tangential trace of the curvature tensor, viewed as a linear transformation of $\mathcal{N}$. The third term $\mathcal{F}^{2}$ in (2.1) is another zeroth order term; it is a linear transformation of the normal space which is quadratic in $\mathcal{F}$. Its action on a normal vector $U^{a^{\prime}}$ is given by

$$
\left(\mathcal{F}^{2} U\right)^{a^{\prime}}=\mathcal{F}_{a b}^{a^{\prime}} \mathcal{F}_{b^{\prime}}^{a b} U^{b^{\prime}}
$$

So $\mathcal{F}^{2}$ is the norm-squared of $\mathcal{F}$ in the tangential indices, viewed as a linear transformation in the normal indices. At each point it is a positive semi-definite transformation of $\mathcal{N}$. 
We apply this discussion to our product situation, taking the background space to be $X \times K$ with its product metric $g_{X}+g_{K}$, and taking the minimal submanifold to be $Z_{0}=N^{\prime} \times \Sigma$. Our perturbation $\delta Z$ is required to satisfy the linearized minimal submanifold equation, which is to say that it must be in the kernel of the Jacobi operator of $Z_{0}$. Corresponding to the product decomposition of our background space, we may write $\delta Z=$ $\left((\delta Z)_{X},(\delta Z)_{K}\right)$, where $(\delta Z)_{X}$ is a normal vector to $N^{\prime}$ (depending on both the point in $N^{\prime}$ and the point in $\Sigma$ ) and $(\delta Z)_{K}$ is a normal vector to $\Sigma$ (depending on both the point in $\Sigma$ and the point in $N^{\prime}$ ). The Jacobi operator of a product minimal submanifold itself has a product decomposition:

$$
\mathcal{J}_{Z_{0}}(\delta Z)=\left(\left(\mathcal{J}_{N^{\prime}}+\mathcal{L}_{\Sigma}\right)(\delta Z)_{X},\left(\mathcal{J}_{\Sigma}+\mathcal{L}_{N^{\prime}}\right)(\delta Z)_{K}\right)
$$

where $\mathcal{L}=-\nabla^{2}$ denotes the scalar Laplacian on the indicated space and $\mathcal{J}$ the Jacobi operator of the indicated minimal submanifold. The operators $\mathcal{L}_{\Sigma}$ and $\mathcal{J}_{\Sigma}$ are self-adjoint elliptic operators on a compact manifold, so each of them has a spectral decomposition with eigenvalues going to $+\infty$. Upon diagonalizing these operators, it is evident that the behavior of $\delta Z$ near the boundary is determined by these eigenvalues and by the form of $\mathcal{L}_{N^{\prime}}$ and $\mathcal{J}_{N^{\prime}}$ near the boundary.

\subsection{Fluctuation in the internal space}

First consider the behavior of $(\delta Z)_{K}$. The equation of motion which follows from $(2.2)$ is

$$
\left(\mathcal{L}_{N^{\prime}}+\lambda\right)(\delta Z)_{K}=0
$$

where $\lambda$ is an eigenvalue for $\mathcal{J}_{\Sigma}$. This is exactly the equation for a massive scalar field on the space $N^{\prime}$ with mass squared given by $\lambda$. Since $N^{\prime}$ intersects the boundary orthogonally, the induced metric is asymptotically hyperbolic, so the near boundary behavior is given by $r^{\Delta_{ \pm}}$with the standard mass/dimension relation

$$
\Delta_{ \pm}=\frac{k}{2} \pm \frac{\sqrt{k^{2}+4 \lambda}}{2}
$$

Our boundary condition requires ${ }^{3} \operatorname{Re} \Delta>0$, so only negative $\lambda$ produce a valid $\Delta_{-}$. There are at most finitely many such $\lambda$, corresponding to the perturbations of $\Sigma$ in directions for which the area decreases. In terms of the holographically dual field theory these fluctuations map to the relevant operators, that is operators whose effect becomes negligible at short distances. When $\lambda$ is very negative; namely $\lambda<-k^{2} / 4, \Delta_{ \pm}$are a complex conjugate pair with real part $k / 2$, corresponding to a scattering phenomenon (when viewing hyperbolic space as the target space of particle motion) or equivalently to an instability (when studying Lorentzian AdS). For $-k^{2} / 4<\lambda<0, \Delta_{ \pm}$are real with $0<\Delta_{-}<k / 2, k / 2<\Delta_{+}<k$. Even though the coefficients of both $r^{\Delta_{-}}$and $r^{\Delta_{+}}$are formally undetermined as functions on

\footnotetext{
${ }^{3}$ In the holographically dual field theory excitations with real and negative $\Delta$ correspond to irrelevant operators. These can not be added to the action as they would spoil the short distance properties of the field theory. We can however add them as sources with delta-function support and calculate correlation functions.
} 
$N$, one anticipates that generically the coefficient of $r^{\Delta_{-}}$(if $\lambda<0$ ) can be chosen arbitrarily but that the coefficients of all of the $r^{\Delta_{+}}$will be determined by global considerations. If $\lambda=$ 0 , then $\Delta_{-}=0$ which violates our boundary condition that $Z$ asymptotically approaches $N \times \Sigma$. However, $\lambda=0$ corresponds to a minimal perturbation of $\Sigma \subset K$, so corresponds to a deformation of $Z_{0}$ in which $\Sigma$ changes and the perturbations $Z$ remain products. In the holographically dual field theory, this maps to what is called a marginal operator.

Negative eigenvalues for $\mathcal{J}_{\Sigma}$ thus play an important role in our analysis because they correspond to additional freedom to prescribe local boundary conditions for minimal submanifolds $Z$. Clearly $\mathcal{J}_{\Sigma}$ depends solely on the geometry of $\Sigma \subset K$, i.e. it is independent of the asymptotically AdS space and its submanifold. Thus the same is true of the $\lambda$ 's. The AdS geometry influences the $\Delta$ 's only through $k$, the dimension of the AdS boundary submanifold. Note that $\mathcal{J}$ scales like (distance) $)^{-2}$, so the $\lambda$ 's scale the same way. In particular, the number of negative $\lambda$ 's is independent of rescaling $g_{K}$. Since $\nabla^{*} \nabla \geq 0$, negative $\lambda$ 's must be created by the influence of $\mathcal{R}$ and $\mathcal{F}^{2}$. Since $\mathcal{F}^{2} \geq 0$, this term always has a negative effect on $\lambda$. The $\mathcal{R}$ term has a negative effect for manifolds of positive sectional curvature and vice versa. One can easily read off some qualitative information from such considerations. For example, if $K$ has non-positive sectional curvature, say a compact hyperbolic manifold or a torus, and $\Sigma$ is totally geodesic (i.e. $\mathcal{F}=0$ ), or even just has sufficiently small extrinsic curvature in the case that $K$ has negative sectional curvature, then $\mathcal{J}_{\Sigma} \geq 0$ so there are no positive $\Delta_{-}$'s and no locally prescribable freedom in the expansion of $(\delta Z)_{K}$. On the other hand, if $K$ has positive curvature and $\Sigma$ is totally geodesic, then we anticipate the possibility of negative $\lambda$ 's and therefore additional freedom for minimal submanifolds $Z$. This freedom will be exhibited in section 4 when $\Sigma$ is an equatorial sphere embedded in a higher-dimensional sphere.

In conclusion, we find that for every eigenvector with negative eigenvalue of the Jacobi operator $\mathcal{J}_{\Sigma}$ associated to the embedding of $\Sigma$ in $K$, there is one piece of local information that needs to be specified at the boundary: the coefficient of $r^{\Delta_{-}}$(as a function on $N$ ). For every eigenvector of the Jacobi operator (even with positive eigenvalue), the coefficient of $r^{\Delta_{+}}$is undetermined by the boundary data and needs to be specified by global considerations. $\Delta_{ \pm}$are given by (2.3).

Fluctuations of the brane which correspond to eigenfunctions of $\mathcal{J}_{\Sigma}$ with positive eigenvalue $\lambda$ encode the spectrum of an infinite tower of relevant operators in the dual field theory whose dimensions are given by $\Delta_{+}$from $(2.3)$.

\subsection{Fluctuation in AdS}

Now consider the behavior of $(\delta Z)_{X}$. The equation of motion reads

$$
\left(\mathcal{J}_{N^{\prime}}+\Lambda\right)(\delta Z)_{X}=0
$$

where $\Lambda$ is an eigenvalue of $\mathcal{L}_{\Sigma}$. We need to determine the leading term in $\mathcal{J}_{N^{\prime}}(\delta Z)_{X}$ under the power law ansatz $(\delta Z)_{X} \sim r^{\Delta}$. Choose coordinates $\left(x^{\alpha}, u^{\alpha^{\prime}}, r\right)$ on $X$ so that $u^{\alpha^{\prime}}$ vanishes on $N^{\prime}$ and $r$ vanishes on $\partial X$. ( $g_{X}$ need not have the form (1.1) in these coordinates.) We use a 0 index to correspond to $r$ and let $\mu, \nu, \sigma$ run over $\alpha$ and 0 and $i, j$, 
$k$ run over all of $\alpha, \alpha^{\prime}, 0$. Let $g$ denote the asymptotically hyperbolic metric $g_{X}$. Using the fact that $N^{\prime}$ intersects $\partial X$ orthogonally, we can arrange that $g^{\mu \alpha^{\prime}}=0$ everywhere on $N^{\prime}$, so that $\mu$ corresponds to directions tangent to $N^{\prime}$ and $\alpha^{\prime}$ to directions normal to $N^{\prime}$. Write $g=r^{-2} \bar{g}$. The asymptotically hyperbolic condition says that $\bar{g}^{00}=1$ on the boundary. The Christoffel symbols of $g$ satisfy

$$
\Gamma_{i j}^{k} \sim-r^{-1}\left(r_{j} \delta_{i}^{k}+r_{i} \delta_{j}^{k}-\bar{g}^{k l} r_{l} \bar{g}_{i j}\right) .
$$

Thus for the $O\left(r^{-1}\right)$ terms we have

$$
\begin{aligned}
\Gamma_{\mu \nu}^{\sigma} & \sim-r^{-1}\left(\delta_{\mu}^{0} \delta_{\nu}^{\sigma}+\delta_{\nu}^{0} \delta_{\mu}^{\sigma}-\bar{g}^{\sigma 0} \bar{g}_{\mu \nu}\right) \\
\Gamma_{\mu \nu}^{\alpha^{\prime}} & \sim 0 \\
\Gamma_{\mu \beta^{\prime}}^{\alpha^{\prime}} & \sim-r^{-1} \delta_{\mu}^{0} \delta_{\beta^{\prime}}^{\alpha^{\prime} .}
\end{aligned}
$$

If $U^{\alpha^{\prime}}$ is a vector field normal to $N^{\prime}$ and smooth up to the boundary, then

$$
\nabla_{\nu}\left(r^{\Delta} U^{\alpha^{\prime}}\right) \sim \Delta r^{\Delta-1} \delta_{\nu}^{0} U^{\alpha^{\prime}}+r^{\Delta} \Gamma_{\nu \beta^{\prime}}^{\alpha^{\prime}} U^{\beta^{\prime}} \sim(\Delta-1) r^{\Delta-1} \delta_{\nu}^{0} U^{\alpha^{\prime}}
$$

so

$$
\begin{aligned}
g^{\mu \nu} \nabla_{\mu} \nabla_{\nu}\left(r^{\Delta} U^{\alpha^{\prime}}\right) & \sim(\Delta-1) g^{\mu \nu} \nabla_{\mu}\left(r^{\Delta-1} \delta_{\nu}^{0} U^{\alpha^{\prime}}\right) \\
& \sim(\Delta-1) g^{\mu \nu}\left[(\Delta-1) r^{\Delta-2} \delta_{\mu}^{0} \delta_{\nu}^{0} U^{\alpha^{\prime}}+r^{\Delta-1} \delta_{\nu}^{0} \Gamma_{\mu \beta^{\prime}}^{\alpha^{\prime}} U^{\beta^{\prime}}-r^{\Delta-1} \Gamma_{\mu \nu}^{\sigma} \delta_{\sigma}^{0} U^{\alpha^{\prime}}\right] \\
& \sim(\Delta-1) g^{\mu \nu} r^{\Delta-2}\left[(\Delta-1) \delta_{\mu}^{0} \delta_{\nu}^{0} U^{\alpha^{\prime}}-\delta_{\mu}^{0} \delta_{\nu}^{0} U^{\alpha^{\prime}}+\left(\delta_{\mu}^{0} \delta_{\nu}^{\sigma}+\delta_{\nu}^{0} \delta_{\mu}^{\sigma}-\bar{g}^{\sigma 0} \bar{g}_{\mu \nu}\right) \delta_{\sigma}^{0} U^{\alpha^{\prime}}\right] \\
& \sim(\Delta-1) r^{\Delta}\left[(\Delta-1) U^{\alpha^{\prime}}-U^{\alpha^{\prime}}+(1-k) U^{\alpha^{\prime}}\right]=(\Delta-1)(\Delta-k-1) U^{\alpha^{\prime}} .
\end{aligned}
$$

The curvature tensor of $g$ satisfies

$$
R^{i j}{ }_{k l} \sim-\left(\delta_{k}^{i} \delta_{l}^{j}-\delta_{l}^{i} \delta_{k}^{j}\right)
$$

so that

$$
R_{\mu \beta^{\prime}}^{\mu \alpha^{\prime}} \sim-(k+1) \delta_{\beta^{\prime}}^{\alpha^{\prime}}
$$

and we have $\mathcal{F}^{2} \sim 0$. Hence

$$
-\mathcal{J}_{N^{\prime}}\left(r^{\Delta} U^{\alpha^{\prime}}\right) \sim[(\Delta-1)(\Delta-k-1)-(k+1)] r^{\Delta} U^{\alpha^{\prime}}=\Delta(\Delta-k-2) r^{\Delta} U^{\alpha^{\prime}} .
$$

We thus obtain $(\delta Z)_{X} \sim r^{\Delta_{ \pm}} U^{\alpha^{\prime}}$ with

$$
\Delta_{ \pm}=\frac{k+2}{2} \pm \frac{\sqrt{(k+2)^{2}+4 \Lambda}}{2} .
$$

For the zero mode $\Lambda=0$ we recover the result of [11] that $\Delta=k+2$ or $\Delta=0$. Just as for internal fluctuations, $\Delta=0$ corresponds to minimal perturbations of $N^{\prime}$. These always exist and there is no constraint on $U^{\alpha^{\prime}}$ at the boundary, corresponding to the freedom to choose the AdS boundary cycle arbitrarily. Since $\Lambda \geq 0$, no other $\Lambda$ 's give a $\Delta_{-}$with $\operatorname{Re} \Delta_{-} \geq 0$, so we have no further freedom to prescribe boundary data. The coefficients of all $\Delta_{+}$are formally undetermined and need to be determined globally. 
In conclusion, we find that there is no local information to be specified except for the boundary submanifold $N$. For every eigenvector of the scalar Laplacian on $\Sigma$, the coefficient of $r^{\Delta_{+}}$is undetermined by the boundary data and needs to be specified by global considerations. $\Delta_{ \pm}$are given by (2.4).

Fluctuations of the submanifold which correspond to eigenfunctions of $\mathcal{L}_{\Sigma}$ with positive eigenvalue $\Lambda$ encode the spectrum of an infinite tower of relevant operators in the dual field theory whose dimensions are given by $\Delta_{+}$from (2.4).

\section{Minimality constraint on internal submanifold}

We saw in section 2.1 that in order that a product submanifold $N^{\prime} \times \Sigma$ be minimal, necessarily the internal factor $\Sigma$ must be minimal in $K$. In this section we show that if $Z$ is a minimal submanifold of $X \times K$, not necessarily a product, with $\partial Z=N \times \Sigma$, then still $\Sigma$ must be minimal in $K$. This result is global in the internal submanifold $\Sigma$. That is, we must assume that $\Sigma$ is a full compact submanifold of $K$, as opposed to just a local piece of one. $Z$ only needs to exist near $N \times \Sigma$, but the result is false in general if $\Sigma$ is only a local piece of a compact submanifold.

The outline of the argument is as follows. Pick arbitrarily a point $p$ of $N$ which remains fixed throughout. For $s \in \Sigma$, the tangent space to $Z$ at $(p, s) \in N \times \Sigma=\partial Z$ is a subspace of $T_{p} X \times T_{s} K$. Its projection to $T_{p} X$ can be written as a graph over $T_{p} N \times \mathbb{R}$, where the $\mathbb{R}$ factor corresponds to the $r$ variable. The "slope" of this graph is a vector $v \in \mathbb{R}^{n-k}$ which depends on $s$. The fact that $Z$ is minimal implies that $v(s)$ satisfies a nonlinear system of partial differential equations as a vector-valued function on $\Sigma$. A direct analysis of this system of equations (integration by parts argument) shows that the only global solution is $v=0$. Geometrically this means that $Z$ intersects the boundary orthogonally in the hyperbolic factor, just like for product minimal submanifolds. Finally, the fact that $v=0$ implies that $N^{\prime} \times \Sigma$ is a product minimal submanifold in $T_{p}^{+} X \times K$, where $T_{p}^{+} X$ denotes the interior half-space in $T_{p} X$ with its induced hyperbolic metric, and $N^{\prime}$ is a vertical plane in $T_{p}^{+} X$. By the result for product minimal submanifolds, we conclude that $\Sigma$ must be minimal in $K$.

We proceed with the details. Choose local coordinates $(x, u, r, s, t)$ as described at the end of section 1 . The $x$ 's restrict to a coordinate system on $N$ and the $s$ 's on $\Sigma$. The $x$ 's may be chosen so that the chosen point $p$ has coordinates $x=0 . Z$ is given by

$$
Z=\{(x, u, r, s, t): u=\varphi(x, r, s), t=\psi(x, r, s)\}
$$

where $\varphi(x, 0, s)=0, \psi(x, 0, s)=0$. Consider the submanifold $Z^{\epsilon}$ obtained from $Z$ by dilating the $(x, u, r)$ coordinates in $X$ by $\epsilon:(\underline{x}, \underline{u}, \underline{r})=\left(\epsilon^{-1} x, \epsilon^{-1} u, \epsilon^{-1} r\right)$, while leaving fixed the coordinates $(s, t)$ in $K$. Thus

$$
Z^{\epsilon}=\left\{(\underline{x}, \underline{u}, \underline{r}, s, t): \underline{u}=\epsilon^{-1} \varphi(\epsilon \underline{x}, \epsilon \underline{r}, s), t=\psi(\epsilon \underline{x}, \epsilon \underline{r}, s)\right\} .
$$

If $g_{X}$ is given by (1.1) with $\bar{g}_{r}=\bar{g}(x, u, r)$, then in the dilated coordinates it is given by $g_{X}^{\epsilon}=\underline{r}^{-2}\left(d \underline{r}^{2}+\bar{g}_{\underline{r}}^{\epsilon}\right)$ with $\bar{g}_{\underline{r}}^{\epsilon}=\bar{g}(\epsilon \underline{x}, \epsilon \underline{u}, \epsilon \underline{r})$. For each $\epsilon, Z^{\epsilon}$ is minimal with respect to 
$g_{X}^{\epsilon}+g_{K}$. Take the limit as $\epsilon \rightarrow 0$. After taking the limit, the variables $(\underline{x}, \underline{u}, \underline{r})$ should be regarded as (infinitesimal) coordinates on the interior half-space $T_{p}^{+} X=\{\underline{r}>0\}$ in the tangent space $T_{p} X$. In particular, they make sense globally: $\underline{x} \in \mathbb{R}^{k}, \underline{u} \in \mathbb{R}^{n-k}, \underline{r}>0$. In the limit we conclude that

$$
Z^{0}=\{(\underline{x}, \underline{u}, \underline{r}, s, t): \underline{u}=\underline{r} v(s), t=0, \underline{r}>0\}
$$

is minimal for the metric $g^{0}+g_{K}$, where $v(s)=\varphi_{r}(0,0, s)$ and

$$
g^{0}=\underline{r}^{-2}\left(d \underline{r}^{2}+\bar{g}(0,0,0)\right)
$$

Now $g^{0}$ is a constant curvature hyperbolic metric on $T_{p}^{+} X$. We have $\partial Z^{0}=T_{p} N \times \Sigma$, where $T_{p} N=\{\underline{u}=0, \underline{r}=0\}$ is a linear subspace in the boundary $\underline{r}=0$ of our hyperbolic space. For each $s \in \Sigma$, the projection of $Z^{0}$ into the hyperbolic factor $\{(\underline{x}, \underline{u}, \underline{r})\}$ is the linear graph $\underline{u}=\underline{r} v(s), \underline{r}>0$ in $T_{p}^{+} X$ with boundary $T_{p} N$. This linear graph varies with $s$, and its "slope" $v(s)$ is a globally defined function on $\Sigma$ with values in $\mathbb{R}^{n-k}$.

By direct analysis of the minimal submanifold equations, we will show below that if $Z^{0}$ given by (3.1) is minimal for $g^{0}+g_{K}$ and if $\Sigma \subset K$ is a compact submanifold, then necessarily $v=0$. Our desired conclusion follows immediately: when $v=0, Z^{0}$ is a product $Z^{0}=N^{\prime} \times \Sigma$, where $N^{\prime}=\{\underline{u}=0\}$ is a vertical plane in hyperbolic space. As we saw in section 2.1, this implies that $\Sigma$ is minimal in $K$. Since $v$ arose as $v=\left.\varphi_{r}\right|_{r=0}$, the statement $v=0$ means exactly that $Z$ intersects the boundary orthogonally in the hyperbolic factor.

In (3.1), $Z^{0}$ is expressed as a graph in the sense that the $(\underline{u}, t)$ variables are given as functions of the $(\underline{x}, \underline{r}, s)$ variables. For simplicity, henceforth we remove the _on $(\underline{x}, \underline{u}, \underline{r})$, relabeling them $(x, u, r)$. The minimal submanifold equation for a graph can be derived by calculating the Euler-Lagrange equation for the area functional. This is carried out, e.g., in $\S 2$ of [11] for a graph expressed as $u=u(x)$ in a space with coordinates $\left(x^{\alpha}, u^{\alpha^{\prime}}\right)$ and background metric $g$. In our application, we will have to replace $x^{\alpha}$ by $\left(x^{\alpha}, r, s^{A}\right)$ and $u^{\alpha^{\prime}}$ by $\left(u^{\alpha^{\prime}}, t^{A^{\prime}}\right)$. For a graph $u=u(x)$, the induced metric $h$ is given in the $x$ coordinates by

$$
h_{\alpha \beta}=g_{\alpha \beta}+2 g_{\alpha^{\prime}(\alpha} u_{, \beta)}^{\alpha^{\prime}}+g_{\alpha^{\prime} \beta^{\prime}} u_{, \alpha}^{\alpha^{\prime}} u_{, \beta}^{\beta^{\prime}},
$$

where the indices after a comma indicate coordinate differentiation. The minimal submanifold equation is:

$\frac{1}{\sqrt{\operatorname{det} h}} \partial_{\beta}\left[\sqrt{\operatorname{det} h} h^{\alpha \beta}\left(g_{\alpha^{\prime} \gamma^{\prime}} u_{, \alpha}^{\alpha^{\prime}}+g_{\alpha \gamma^{\prime}}\right)\right]-\frac{1}{2} h^{\alpha \beta}\left[g_{\alpha \beta, \gamma^{\prime}}+2 g_{\alpha \alpha^{\prime}, \gamma^{\prime}} u_{, \beta}^{\alpha^{\prime}}+g_{\alpha^{\prime} \beta^{\prime}, \gamma^{\prime}} u_{, \alpha}^{\alpha^{\prime}} u_{, \beta}^{\beta^{\prime}}\right]=0$.

Now $\alpha$ must be replaced by $(\alpha, r, A)$ and $\alpha^{\prime}$ by $\left(\alpha^{\prime}, A^{\prime}\right)$, and the background metric is $g=g^{0}+g_{K}$, where $g^{0}$ is given by (3.2). By (1.2), we can write $\bar{g}(0,0,0)=\bar{g}_{\alpha \beta} d x^{\alpha} d x^{\beta}+$ $\bar{g}_{\alpha^{\prime} \beta^{\prime}} d u^{\alpha^{\prime}} d u^{\beta^{\prime}}$, where $\bar{g}_{\alpha \beta}$ and $\bar{g}_{\alpha^{\prime} \beta^{\prime}}$ are constant. Equation (3.4) breaks into two sets of equations corresponding to the decomposition of the primed variables as $\left(\gamma^{\prime}, C^{\prime}\right)$. Restrict consideration to the equations labeled by $\gamma^{\prime}$, corresponding to the variables $u^{\gamma^{\prime}}$. In (3.4), all terms in the second brackets [ ] vanish since all coefficients of $g$ are independent of $u^{\gamma^{\prime}}$. Moreover, the term $g_{\alpha \gamma^{\prime}}$ vanishes since $g$ has no nontrivial components $g_{\alpha \gamma^{\prime}}, g_{r \gamma^{\prime}}, g_{A \gamma^{\prime}}$. 
Since $g_{\alpha^{\prime} \gamma^{\prime}}=r^{-2} \bar{g}_{\alpha^{\prime} \gamma^{\prime}}$, we conclude that the $\gamma^{\prime}$ piece of (3.4) reduces to

$$
(\sqrt{\operatorname{det} h})^{-1} \partial_{\beta}\left[r^{-2} \sqrt{\operatorname{det} h} h^{\alpha \beta} \partial_{\alpha} u^{\alpha^{\prime}}\right]=0,
$$

where $\alpha, \beta$ still represent triples $(\alpha, r, A),(\beta, r, B)$.

According to (3.1) and (3.3), we have

$$
h=\left(\begin{array}{ccc}
r^{-2} \bar{g}_{\alpha \beta} & 0 & 0 \\
0 & r^{-2}\left(1+|v|^{2}\right) & r^{-1}\left\langle v, v_{, B}\right\rangle \\
0 & r^{-1}\langle v, A, v\rangle & g_{A B}+\left\langle v_{, A}, v_{, B}\right\rangle
\end{array}\right) .
$$

Here $|\cdot|$ and $\langle\cdot, \cdot\rangle$ denote the norm and inner product on $\mathbb{R}^{n-k}$ determined by $\bar{g}_{\alpha^{\prime} \beta^{\prime}}, v_{, A}$ denotes $\partial_{s^{A}} v, g_{A B}$ is evaluated at $(s, t=0)$, and the blocks correspond to the decomposition $(\alpha, r, A)$. Denote by $H$ the $(1+l) \times(1+l)$ matrix $($ recall $l=\operatorname{dim} \Sigma)$ :

$$
H=\left(\begin{array}{cc}
H_{r r} & H_{r B} \\
H_{A r} & H_{A B}
\end{array}\right)=\left(\begin{array}{cc}
1+|v|^{2} & \left\langle v, v_{, B}\right\rangle \\
\left\langle v_{, A}, v\right\rangle & g_{A B}+\left\langle v_{, A}, v_{, B}\right\rangle
\end{array}\right) .
$$

It is evident that $H$ is positive definite, since

$$
H=\left(\begin{array}{cc}
1 & 0 \\
0 & g_{A B}
\end{array}\right)+\left(\begin{array}{cc}
|v|^{2} & \left\langle v, v_{, B}\right\rangle \\
\left\langle v_{, A}, v\right\rangle & \left\langle v_{, A}, v_{, B}\right\rangle
\end{array}\right)
$$

is the sum of a positive definite matrix and a positive semidefinite matrix. For a given function $v: \Sigma \rightarrow \mathbb{R}^{n-k}, H$ can be interpreted as a metric on $\mathbb{R} \times \Sigma$ which is translationinvariant in the $r$ direction. Now $\sqrt{\operatorname{det} h}=r^{-k-1} \sqrt{\operatorname{det} \bar{g}_{\alpha \beta}} \sqrt{\operatorname{det} H}$. Thus (3.5) becomes

$$
(\sqrt{\operatorname{det} H})^{-1} \partial_{\beta}\left[r^{-k-3} \sqrt{\operatorname{det} H} h^{\alpha \beta} \partial_{\alpha} u^{\alpha^{\prime}}\right]=0 .
$$

Since $u^{\alpha^{\prime}}=r v^{\alpha^{\prime}}(s)$, only the $(r, A)$ pieces of $\alpha$ enter into the summation. The left-hand side of (3.6) is of the form $r^{-k-2}$ times a function of $s$ alone. This function of $s$ can be identified by expanding the $\alpha, \beta$ sums. One finds that (3.6) is equivalent to:

$$
(\sqrt{\operatorname{det} H})^{-1} \partial_{B}\left[\sqrt{\operatorname{det} H}\left(H^{A B} v_{, A}^{\alpha^{\prime}}+H^{r B} v^{\alpha^{\prime}}\right)\right]-(k+1)\left(H^{A r} v_{, A}^{\alpha^{\prime}}+H^{r r} v^{\alpha^{\prime}}\right)=0,
$$

where we write

$$
H^{-1}=\left(\begin{array}{ll}
H^{r r} & H^{r B} \\
H^{A r} & H^{A B}
\end{array}\right) .
$$

Equation (3.7) exhibits concretely the partial differential equation on $v^{\alpha^{\prime}}(s)$ implied by the condition that $Z^{0}$ is minimal for $g^{0}+g_{K}$.

We now show that $v^{\alpha^{\prime}}=0$ is the only global solution of (3.7) on a compact manifold $\Sigma$ with metric $g_{A B}$. Since $\sqrt{\operatorname{det} H}$ defines a volume form on $\mathbb{R} \times \Sigma$ which is independent of $r$, it can also be interpreted as a volume form on $\Sigma$ itself. Suppose $v^{\alpha^{\prime}}$ solves (3.7). Multiply (3.7) by $\bar{g}_{\alpha^{\prime} \beta^{\prime}} v^{\beta^{\prime}} \sqrt{\operatorname{det} H}$ and integrate by parts over $\Sigma$. This gives

$$
\int_{\Sigma}\left[H^{A B}\left\langle v_{, A}, v_{, B}\right\rangle+(k+2) H^{A r}\left\langle v_{, A}, v\right\rangle+(k+1) H^{r r}|v|^{2}\right] \sqrt{\operatorname{det} H} d s=0 .
$$


Decompose the integrand as

$$
\left(H^{A B}\left\langle v_{, A}, v_{, B}\right\rangle+2 H^{A r}\left\langle v_{, A}, v\right\rangle+H^{r r}\langle v, v\rangle\right)+k\left(H^{A r}\left\langle v_{, A}, v\right\rangle+H^{r r}|v|^{2}\right) .
$$

Since $H^{-1}$ is positive definite, the first term is nonnegative. So if we can show that

$$
H^{r r}|v|^{2}+H^{A r}\left\langle v_{, A}, v\right\rangle \geq 0
$$

then both terms separately must vanish. From the vanishing of the first term we can conclude that $v^{\alpha^{\prime}}=0$ as desired.

It remains to establish (3.8). Let

$$
\left(\begin{array}{ll}
C^{r r} & C^{r B} \\
C^{A r} & C^{A B}
\end{array}\right)
$$

denote the cofactor matrix of $H$, so that

$$
\operatorname{det} H=H_{r r} C^{r r}+H_{A r} C^{A r}
$$

and

$$
\left(\begin{array}{ll}
H^{r r} & H^{r B} \\
H^{A r} & H^{A B}
\end{array}\right)=(\operatorname{det} H)^{-1}\left(\begin{array}{ll}
C^{r r} & C^{r B} \\
C^{A r} & C^{A B}
\end{array}\right) .
$$

In particular, $H^{r r}|v|^{2}+H^{A r}\left\langle v_{, A}, v\right\rangle=(\operatorname{det} H)^{-1}\left(C^{r r}|v|^{2}+C^{A r}\left\langle v_{, A}, v\right\rangle\right)$. But the cofactor expansion along the first column gives

$$
C^{r r}|v|^{2}+C^{A r}\left\langle v_{, A}, v\right\rangle=\operatorname{det}\left(\begin{array}{ll}
|v|^{2} & H_{r B} \\
\left\langle v_{, A}, v\right\rangle & H_{A B}
\end{array}\right)=\operatorname{det}\left(\begin{array}{ll}
|v|^{2} & \left\langle v, v_{, B}\right\rangle \\
\left\langle v_{, A}, v\right\rangle & g_{A B}+\left\langle v_{, A}, v_{, B}\right\rangle
\end{array}\right) .
$$

The matrix

$$
\left(\begin{array}{ll}
|v|^{2} & \left\langle v, v_{, B}\right\rangle \\
\left\langle v_{, A}, v\right\rangle & g_{A B}+\left\langle v_{, A}, v_{, B}\right\rangle
\end{array}\right)
$$

is positive semidefinite for the same reason that $H$ was positive definite: it can be written as the sum

$$
\left(\begin{array}{ll}
0 & 0 \\
0 & g_{A B}
\end{array}\right)+\left(\begin{array}{ll}
|v|^{2} & \langle v, v, B\rangle \\
\left\langle v_{, A}, v\right\rangle & \left\langle v_{, A}, v, B\right.
\end{array}\right)
$$

of two positive semidefinite matrices. Therefore its determinant is nonnegative. This establishes (3.8) and so concludes the argument.

We remark that the vanishing of $v^{\alpha^{\prime}}$ can alternately be proved by an integration by parts argument on $\mathbb{R} \times \Sigma$ rather than on $\Sigma$. Namely, multiply (3.6) by $g_{\alpha^{\prime} \beta^{\prime}} u^{\beta^{\prime}} \sqrt{\operatorname{det} H} d s d r$, integrate $r$ over $(a, b)$ and $s$ over $\Sigma$, where $0<a<b<\infty$ are fixed, and then integrate by parts in both $r$ and $s$. The $r$ integration gives rise to a boundary term, but (3.8) implies that it has a sign.

For analytic metrics $g_{A B}$, nonzero local solutions $v^{\alpha^{\prime}}$ of (3.7) can be constructed as convergent power series (Cauchy-Kowalewski Theorem). By appropriately choosing the background metric $g_{K}$, it can be arranged that the submanifold $Z^{0}$ given by (3.1) for such a solution $v^{\alpha^{\prime}}$ is minimal with respect to $g^{0}+g_{K}$ while at the same time $\Sigma$ is not minimal with respect to $g_{K}$. Such a $Z^{0}$ thus provides a local example of a minimal submanifold of a product space $X \times K$ whose boundary is a product $N \times \Sigma$ with $\Sigma$ not minimal with respect to $g_{K}$. 


\section{Examples}

\subsection{Slipping modes on spheres}

\subsubsection{The flavor D7 brane}

Let us begin by reviewing one example in which a " $t$ " variable is a non-trivial function of $r$ as first presented in [9]. The submanifold fills all of $X$, which for simplicity we take to be (Euclidean) $A d S_{5}$ parametrized in standard Poincaré coordinates

$$
d s_{\text {AdS }}^{2}=\frac{1}{r^{2}}\left(d r^{2}+d \vec{x}^{2}\right) .
$$

$\vec{x}$ are Cartesian coordinates along the $\mathbb{R}^{4}$ factor. Let us take the internal space to be $S^{5}$ with metric written as

$$
d s^{2}=d \theta^{2}+\cos ^{2}(\theta) d \Omega_{3}^{2}+\sin ^{2}(\theta) d \psi^{2}
$$

where $d \Omega_{3}^{2}$ is the round metric on the 3 -sphere.

We are looking for a submanifold of the form

$$
\psi=\text { const. }, \quad \theta=\theta(r)
$$

with $\theta(0)=0$. This defines an 8-dimensional submanifold of the 10 dimensional product space. Asymptotically, the submanifold fills all of $A d S_{5}(k=n=4)$ and it wraps an equatorial $S^{3}$ inside $S^{5}(l=3)$. This submanifold is the worldvolume of a D7 brane, where the standard physics nomenclature defines a $\mathrm{D} p$ brane as an object extended in $p$ spatial dimensions and hence with a $p+1$ dimensional worldvolume.

The ansatz describes a submanifold where the $S^{3}$ wrapped by the D7 shrinks as a function of the radial coordinate: the D7 "slips off". Correspondingly, $\theta$ is often referred to as the "slipping mode" in the physics literature. If $\theta(r)$ reaches $\pi / 2$ at any finite $r$, the internal sphere shrinks to zero size at that point. For a generic submanifold of this type there will be a singularity at this point. Imposing regularity as an additional constraint results in a unique submanifold for given local data on the boundary.

To find the solution, one starts with the area functional restricted to this ansatz:

$$
\mathcal{A}=r^{-5} \cos ^{3}(\theta) \sqrt{1+r^{2}\left(\theta^{\prime}\right)^{2}}
$$

and treats it as the Lagrangian of a classical mechanics problem. The resulting EulerLagrange equation gives a non-linear 2nd order ordinary differential equation for $\theta(r)$. Nevertheless, it is easy to verify that

$$
\theta=\arcsin (m r)
$$

where $m$ is a constant, defines a one parameter family of solutions. ${ }^{4}$ For this solution, we can expand near the boundary

$$
\theta \sim m r-\frac{(m r)^{3}}{6}+\mathcal{O}\left(r^{5}\right)
$$

\footnotetext{
${ }^{4}$ The reason that such a simple solution exists is supersymmetry. Instead of solving the 2nd order differential equations directly one can find this arcsin solution by solving an auxiliary problem of finding a particular Killing spinor, which amounts to solving a first order equation. See [9].
} 
The local data is the coefficient $m$ of $r^{\Delta_{-}}=r$. The global data is the coefficient $-m^{3} / 6$ of $r^{\Delta_{+}}=r^{3}$; for this solution it is indeed fixed in terms of the local data. The submanifold does not extend past $r=1 / m$. At $r=1 / m$ the submanifold terminates smoothly. The 3 -sphere it is wrapping inside the 5 -sphere shrinks to zero size, but locally the induced metric just becomes flat $\mathbb{R}^{8}$ and there is no curvature singularity.

The physical interpretation of this flavor brane is as outlined in the introduction. It adds fundamental representation matter (in this case a hypermultiplet preserving 8 supercharges) to the field theory which otherwise only hosts adjoint representation fields $(\mathcal{N}=4 \mathrm{SYM}$ in this case). The "slipping mode" $\theta(r)$ in the field theory maps to a bi-linear operator made from two defect fields. Turning on the coefficient of $r^{\Delta_{-}}=r$ corresponds in the field theory to adding a mass for the flavors. The coefficient of $r^{\Delta_{+}}=r^{3}$ is related to the vacuum expectation value of this bi-linear operator, the "chiral condensate"; the precise relation has been worked out e.g. in [12] using the technique of holographic renormalization (holo-RG). The regularity condition in the interior (the IR boundary condition) fixes the relation between the two; the arcsin solution is determined by a single parameter $m$.

\subsubsection{General AdS times sphere example}

The example of the previous subsection can easily be generalized to submanifolds that asymptote to $\mathrm{AdS}_{k+1} \times S^{l}$ inside $\mathrm{AdS}_{n+1} \times S^{m}$. Now we write

$$
d s_{\mathrm{AdS}}^{2}=\frac{1}{r^{2}}\left(d r^{2}+d \vec{x}^{2}+d \vec{y}^{2}\right)
$$

with $\vec{x} \in \mathbb{R}^{k}$ and $\vec{y} \in \mathbb{R}^{n-k}$. As above, write the metric on $S^{m}$ as

$$
d s^{2}=d \theta^{2}+\cos ^{2}(\theta) d \Omega_{l}^{2}+\sin ^{2}(\theta) d \Omega_{m-l-1}^{2} .
$$

To keep the discussion general, we also allow for a slightly more general background metric: we study submanifolds in $\operatorname{AdS}_{n+1} \times S^{m}$ where we allow the radius of curvature $R_{s}$ of the internal sphere $S^{m}$ to be different from the radius of curvature $R=1$ of $\operatorname{AdS}_{n+1}$, with $\left(R_{s} / R\right)^{2}=\alpha$. The $\vec{y}$ variables and the variables in $S^{m-l-1}$ are constant on the submanifolds under consideration so these variables will play no role.

One possible minimal area submanifold in all these cases is the trivial $(k+1+l)$ dimensional submanifold $\theta=0$, which is globally $\operatorname{AdS}_{k+1} \times S^{l}$, not just asymptotically close to the boundary. The Lagrangian (the area element) for the slipping mode $\theta(r)$ is

$$
\mathcal{A}=r^{-k-1} \cos ^{l}(\theta) \sqrt{1+\alpha r^{2}\left(\theta^{\prime}\right)^{2}} .
$$

While $\theta=0$ is a solution, we can deform the cycle by turning on $\theta$ and only requiring that $\theta$ go to zero asymptotically. Close to the boundary we can linearize the equations in $\theta$. The slipping mode acts like a scalar field with mass squared $M^{2}=-l / \alpha$ in $\mathrm{AdS}_{k+1}$. Its near boundary behavior is $r^{\Delta}$ with the usual

$$
\Delta_{ \pm}=\frac{k}{2} \pm \frac{1}{2} \sqrt{k^{2}+4 M^{2}}=\frac{k}{2} \pm \frac{1}{2} \sqrt{k^{2}-4 l / \alpha} .
$$

$\Delta$ becomes complex for $\alpha<\alpha_{\text {crit }}=4 l / k^{2}$. 
For the D7 example above $\alpha=1, k=4$ and $l=3$ so $\Delta=1$ and $\Delta=3$ are the two solutions. A similarly nice example [14] is the D5 brane with $\mathrm{AdS}_{4} \times S^{2}$ asymptotics in $\mathrm{AdS}_{5} \times S^{5}$. Then $\alpha=1, k=3$ and $l=2$, so that $\Delta=1$ and $\Delta=2$ are the two solutions. In this case the arcsine of (4.1) is once more an analytic solution. In fact, it is easy to check that (4.1) solves the equations of motion whenever $\alpha=1$ and $k=l+1$. The corresponding dimensions $\Delta$ again turn out to be integers in this case, $\Delta=1$ and $\Delta=k-1$. But in general $\Delta$ is irrational. If one in addition takes the slipping mode to depend on the $S^{l}$ coordinates, one finds solutions (to the linearized equations) where $\theta$ is a spherical harmonic (eigenfunction of the Laplacian with eigenvalue $L(L+l-1)$ ) on the internal space and its effective mass squared in AdS is then

$$
\alpha M^{2}=-l+L(L+l-1)
$$

For $\alpha=1$ and $l=k-1$ this gives $\Delta=1-L$ and $\Delta=k-1+L$, and in particular for the D7 example one has $\Delta=1-L$ and $\Delta=3+L$.

The two different $\Delta$ 's have standard interpretation in the physics literature. The smaller $\Delta$ corresponds to a non-normalizable mode. Turning it on amounts to deforming the theory. This way one naturally obtains a one-parameter family of submanifolds. This is exactly the parameter $m$ in the D7 example before. Only if the mass squared lies between $-k^{2} / 4$ and 0 does one get a positive $\Delta$ for this non-normalizable mode. The larger value of $\Delta$ corresponds to the normalizable mode. As in the D7 example, its coefficient is usually fixed by a regularity condition in the interior. If the mass squared is positive we get one positive and one negative $\Delta$. So if we want the submanifold to be regular at $r=0$, in this case one is limited to the larger (normalizable) $\Delta$. In the physics language the positive mass squared corresponds to "irrelevant" operators. When added to the field theory lagrangian (= turn on the leading behavior of the scalar) they grow at high energies (=close to the boundary); their backreaction destroys the AdS asymptotics and so we do not consider them here. They can still have a non-trivial expectation value (= coefficient of the subleading term) which is determined dynamically (=by a regularity condition in the interior).

In terms of physics, the interpretation of the more general flavor branes discussed in this subsection is very similar to the D7 example above. Some fundamental representation matter is added to the field theory. Of course for $\alpha \neq 1$ and general $k$ and $l$, we don't have a known duality that realizes this background/minimal area pair. If $k<n$, the flavor is localized on a defect, for $k=n$ it is spacetime-filling as in the D7 case. In all these cases, one expects a single regular solution to exist for a given coefficient of the $r^{\Delta_{-}}$asymptotic term. The simple analytic solution in terms of an arcsin is explained by supersymmetry for the D7 and the D5 examples above.

In (2.1) we gave a general formula for the Jacobi operator. Let us confirm that this reproduces the same results for these examples. An equatorial $S^{l} \subset S^{m}$ is totally geodesic, so $\mathcal{F}=0$, and one sees easily that $\mathcal{R}=l$ Id (unless $m=1$, in which case $\mathcal{R}=0$ even if $l=1)$. The normal bundle is trivial with a global parallel frame. So the spectrum of $\nabla^{*} \nabla$ on $\mathcal{N}$ consists of the spectrum of the scalar Laplacian on $S^{l}$, namely $\{L(L+l-1)\}$, with multiplicity $m-l$. For $\alpha=1$ this gives $\lambda=L(L+l-1)-l$ and is consistent with the 
discussion above. The rescaling observation in section 2.3 produces the correct dependence on $\alpha$ in (4.3).

Note that $\lambda=0$ for $L=1$, so there is exactly one negative $\lambda$, and that this persists under general rescaling of $g_{K}$. Thus the choice $L=0$ is the only possibility to obtain $\operatorname{Re} \Delta_{-}>0$, i.e. we can only have one relevant deformation for any $S^{l} \subset S^{m}$ and any scaling of $g_{K}$.

\subsection{Disjoint boundaries}

\subsubsection{Wilson lines with internal motion}

An example with both " $t$ " and " $u$ " variables being non-trivial functions of $r$ is the Wilson line of [7]. In this case the boundary is disconnected with two components: $\partial Z=\left(N_{1} \times \Sigma_{1}\right) \cup$ $\left(N_{2} \times \Sigma_{2}\right)$. Generalizations of this example will allow us to study in detail the backreaction of the " $u$ "-variables on the " $t$ "-variables and vice versa. For the case of the rectangular Wilson line in $\mathrm{AdS}_{5} \times S^{5}$ discussed in [7], $N_{1,2}$ are parallel straight lines separated by a distance $\Delta u$ and $\Sigma_{1,2}$ are points separated by a distance $\Delta \theta$ on the internal sphere. Locally, close to each of the components of the boundary, the minimal area asymptotes to $\mathrm{AdS}_{2}$ times a point, but globally the two asymptotic regions are connected into one smooth U-shaped minimal surface. The special case $\Delta \theta=0$ corresponds simply to a minimal surface in $\mathrm{AdS}_{5}$. A second example $[12,15,16]$ has a D5 brane with two locally $A d S_{4} \times S^{2}$ asymptotic regions ending on two parallel $\mathbb{R}^{3} \times S^{2}$ boundaries, where the $S^{2}$ in both cases is the same equatorial $S^{2}$ inside the $S^{5}$. The equatorial $S^{2}$ does not move: this example is a product of a four-dimensional minimal submanifold of $\mathrm{AdS}_{5}$ with $S^{2}$. All of these examples fit into the more general framework of having $\partial Z$ be two copies of $\mathbb{R}^{k} \times S^{l}$ for which the two $\mathbb{R}^{k}$ 's are parallel. In the next two subsections we consider two different generalizations of these examples to $\mathbb{R}^{k} \times S^{l}$ :

- The most direct generalization of the example of [7] has two disjoint copies of $\mathbb{R}^{k} \times S^{l}$ where the background internal space is $S^{2 l+1}$ and the two $S^{l}$ 's are equatorial and disjoint. They still can be connected by a smooth U shaped submanifold. This set of examples in particular contains the Wilson line with separate points on the internal sphere as the special case of $l=0$.

- The second generalization has two disjoint copies of $\mathbb{R}^{k} \times S^{l}$ with the same equatorial $S^{l}$ for both, as in the D5 example above. But now we turn on a non-trivial slipping mode on each of the disjoint defects, leading to an interesting interplay between internal and AdS coordinates.

The physics interpretation of a single such defect was discussed in the previous subsection: each defect adds fundamental matter to the gauge theory, localized on $N_{1}$ and $N_{2}$ respectively. For a connected worldvolume in the bulk to be allowed, one needs one of the defects to be an anti-defect. Flavor D-branes come with an orientation, and in the connected worldvolume the orientation changes between the two defects. This was already the case in the Wilson line example, where the U-shaped worldvolume evaluates 
the quark/anti-quark potential, not the quark-quark potential. For the latter only a disconnected worldvolume is allowed in the bulk. For quark/anti-quark both connected and disconnected configurations are allowed (and often there is a competition between the two for which one has the lower area). Such brane/anti-brane configurations typically break all supersymmetry. In the D5 example, both D5 and anti-D5 individually preserve half the supersymmetry, but it is the opposite half that is preserved. Together they break supersymmetry completely.

\subsubsection{Rotating spheres}

Let us first look at a case of two disjoint copies of $\mathbb{R}^{k} \times S^{l}$, where the background internal space is $S^{2 l+1}$ and the two $S^{l}$ 's are equatorial and disjoint. We write

$$
d s_{\mathrm{AdS}}^{2}=\frac{1}{r^{2}}\left(d r^{2}+d \vec{x}^{2}+d u^{2}+d \vec{y}^{2}\right)
$$

with $\vec{x} \in \mathbb{R}^{k}, u \in \mathbb{R}, \vec{y} \in \mathbb{R}^{n-k-1}$. Once again $\vec{y}$ plays no role. We are looking for a maximally symmetric solution which is translation-invariant in $\vec{x}$ and where $u(r)$ is turned on as the only " $u$ " variable. Asymptotically, $u(r)$ should be a double-valued function which approaches $u(0)= \pm \Delta u / 2$. The two branches will be smoothly connected at a turning point at $r_{\max }$ with $u(r) \sim \sqrt{r_{\max }-r}$ close to $r_{\max }$.

To get the Lagrangian, we write the $S^{2 l+1}$ metric as

$$
d s^{2}=d \theta^{2}+\cos ^{2}(\theta) d S_{l}^{2}+\sin ^{2}(\theta) d \tilde{S}_{l}^{2} .
$$

We embed $S^{l} \times S^{1}$ into $S^{2 l+1} \subset \mathbb{R}^{2 l+2}$ by

$$
(w, \theta) \rightarrow(\cos (\theta) w, \sin (\theta) w)
$$

where $w \in \mathbb{R}^{l+1},|w|=1$. Then the $S^{2 l+1}$ metric pulls back to just

$$
d s^{2}=d \theta^{2}+d S_{l}^{2}(w)
$$

Adding this to the usual AdS metric and then taking $u$ and $\theta$ to be functions of $r$ as before, the $d S_{l}^{2}(w)$ does not interact with anything in forming the Lagrangian, and the effective Lagrangian is independent of $l$ :

$$
\mathcal{A}=r^{-k-1} \sqrt{1+\left(u^{\prime}\right)^{2}+\alpha r^{2}\left(\theta^{\prime}\right)^{2}} .
$$

As a result, the submanifold for any $l$ is just given by the generalization of the solution in [7] from $k=1$ and $\alpha=1$ to arbitrary $k$ and $\alpha$, but independent of $l$. This solution is derived by observing that $u$ and $\theta$ do not appear in $\mathcal{A}$, so there are two conserved quantities. This leads to

$$
u^{\prime}= \pm c_{1} \frac{r^{k+1}}{\sqrt{1-c_{1}^{2} r^{2 k+2}-\alpha c_{2}^{2} r^{2 k}}}, \quad \theta^{\prime}=\frac{c_{2}}{c_{1} r^{2}} u^{\prime}
$$

from which $u$ and $\theta$ are obtained by integration. The two integration constants $c_{1}$ and $c_{2}$ set the separations $\Delta u$ and $\Delta \theta$ of the two disjoint boundary pieces. The case $c_{2}=0$ 
corresponds to a product solution with a U-shaped minimal submanifold of hyperbolic space. The remaining two integration constants set the overall position. They can always be set to zero by exploiting translation invariance in $u$ and $\theta$.

Let us briefly see how these exact solutions of the full non-linear system fit into our general description. As we noted at the end of section 4.1.2, we get that $\alpha M^{2}=0$ for $L=1$ for any dimension $l$ of the internal sphere (and for any $\alpha$ ). By locking the two $S^{l}$ spheres to each other, we implicitly turned on an $L=1$ mode. With this, we get $\Delta_{-}=0$ and $\Delta_{+}=k$ for the $\theta$ mode, which is consistent with the solution (4.5).

From the physics point of view it is somewhat surprising why the $L=1$ mode is so special in the sense that a simple solution to the full non-linear equations can be found for any $l$. Typically the slipping mode describes a bi-linear operator made out of two defect fields, whereas $L=1$ describes a tri-linear operator made of two defect fields and one of the adjoint fields. The dual solution has the brane rotating inside the internal $S^{2 l+1}$ along the U-shaped worldvolume.

\subsubsection{Interaction between internal and hyperbolic factors}

For $l \geq 1$ we can consider a second class of examples, where this time the two disjoint asymptotic defects wrap the same $S^{l}$, but we turn on a slipping mode. As before, asymptotically the slipping mode will scale ${ }^{5}$ as $\theta \sim r^{\Delta_{-}}$. The coefficient of this $r^{\Delta_{-}}$does not have to be the same on the two disjoint defects.

We write the AdS metric in the form (4.4) and the $S^{m}$ metric in the form (4.2), with an overall prefactor of $\alpha$ for the metric on $S^{m}$ to account for the difference in curvature radii. The $\vec{y}$ variables and the variables in $S^{m-l-1}$ are held constant. We are interested in solutions where we turn on the slipping mode $\theta(r)$ as our only " $t$ " variable, and $u(r)$ as our only " $u$ " variable. This ansatz is forced upon us if we insist on preserving the full symmetry of $\mathbb{R}^{k} \times S^{l}$ as well as the isometries of the transverse $S^{m-l-1}$. With this ansatz, the Lagrangian for the area of the submanifold parametrized by $\theta(r)$ and $u(r)$ is proportional to

$$
\mathcal{A}=\frac{\cos ^{l}(\theta)}{r^{k+1}} \sqrt{1+\left(u^{\prime}\right)^{2}+\alpha r^{2}\left(\theta^{\prime}\right)^{2}}
$$

As $u$ only appears derivatively (as a consequence of translation invariance of the background metric) we can solve for $u(r)$ explicitly using an integral of motion:

$$
c=\frac{\delta \mathcal{A}}{\delta u^{\prime}}=\frac{\cos ^{l}(\theta)}{r^{k+1}} \frac{u^{\prime}}{\sqrt{1+\left(u^{\prime}\right)^{2}+\alpha r^{2}\left(\theta^{\prime}\right)^{2}}}
$$

which is easily solved for $u^{\prime}$

$$
u^{\prime}= \pm c r^{k+1} \frac{\sqrt{1+\alpha r^{2}\left(\theta^{\prime}\right)^{2}}}{\sqrt{\cos ^{2 l}(\theta)-c^{2} r^{2 k+2}}}
$$

\footnotetext{
${ }^{5}$ For $l=0$ one has $\Delta_{-}=0$ so turning on the non-normalizable piece of the slipping mode has in this case the interpretation of actually separating the points and so is identical to the case discussed in the previous subsection.
} 
The equations are quadratic in $u^{\prime}$, so we get a free sign choice. The two allowed choices correspond to the two branches. To obtain the equations of motion of $\theta(r)$ we want to substitute $u^{\prime}(r)$ back into the original Lagrangian. One has to be careful though that, while the original Lagrangian instructed us to vary with respect to $\theta$ at fixed $u$ and $u^{\prime}$, we now want to keep $c=\delta \mathcal{A} / \delta u^{\prime}$ fixed, that is the conjugate momentum. The correct action from which to derive the equation of motion for $\theta(r)$ by varying with respect to $\theta$ at fixed $c$ is the Legendre transformed

$$
\tilde{\mathcal{A}}=\mathcal{A}-u^{\prime} \frac{\delta \mathcal{A}}{\delta u^{\prime}}=\frac{1}{r^{k+1}} \sqrt{1+\alpha r^{2}\left(\theta^{\prime}\right)^{2}} \sqrt{\cos ^{2 l}(\theta)-c^{2} r^{2 k+2}} .
$$

In the special case $l=0$ this $\tilde{\mathcal{A}}$ only depends on $\theta^{\prime}$, not on $\theta$, and the full system can be solved analytically as in section 4.2.2. For general $l$ one has to resort to numerics to construct $\theta(r)$, as has recently been carried out in [17] for $k=3, l=2$.

For this family of examples we can study higher order terms in the near boundary expansion analytically. All we need to do is inspect our explicit solution for $u^{\prime}$ as well as the form of the effective action for $\theta$. Let us first look at the asymptotic form of $\theta(r)$. Close to the boundary $\theta(r)$ vanishes and we can determine its behavior by expanding $\tilde{\mathcal{A}}$ to quadratic order in $\theta$ as well as in $r$. Dropping irrelevant $\theta$ independent terms we get

$$
\tilde{\mathcal{A}} \sim \frac{1}{2 r^{k+1}}\left(\alpha r^{2}\left(\theta^{\prime}\right)^{2}\left(1-\frac{1}{2} c^{2} r^{2 k+2}+\ldots\right)-l \theta^{2}\right) .
$$

To find the leading near boundary behavior we can neglect the subleading $c^{2} r^{2 k+2}$ term, which gives us the backreaction of $u$ on $\theta$. The remaining action is just the one of a scalar field of mass squared $M^{2}=-l / \alpha$ in $\mathrm{AdS}_{k+1}$. Correspondingly, the two possible boundary behaviors are once more

$$
\Delta_{ \pm}=\frac{k}{2} \pm \frac{1}{2} \sqrt{k^{2}-4 l / \alpha} .
$$

Armed with our knowledge about $\theta(r)$ we can inspect formula (4.6) for $u^{\prime}(r)$ to determine the leading near boundary behavior of $u$. Since $\theta$ goes to zero at the boundary, the leading small $r$ behavior of $u^{\prime}$ is given by $u^{\prime} \sim c r^{k+1}$ and so $c$ represents the locally undetermined coefficient in the expansion of $u$. It affects $u$ at order $r^{k+2}$ as expected. $c$ is determined in terms of $\Delta u$ for the connected configuration. The leading correction due to the backreaction of $\theta$ comes from the $c \alpha r^{k+3}\left(\theta^{\prime}\right)^{2}$ and $c r^{k+1} \theta^{2}$ corrections to $u^{\prime}$ which arise from expanding out the square roots and the $\cos ^{2 l}(\theta)$ term. This affects $u$ itself at order $r^{k+2+2 \Delta_{-}}$. (We are thinking of the situation $k^{2}>4 l / \alpha$ so that $\Delta_{ \pm}$are real with $\Delta_{-}<\Delta_{+}$.) Last but not least, we need to understand the backreaction of $u$ on $\theta$. This backreaction is determined by the term proportional to $c^{2}$ in the action (4.7) for the $\theta$ fluctuations. Again droppping $\theta$ independent terms, we can write (4.7) as

$$
\tilde{\mathcal{A}} \sim r^{-k-1}\left[\cos ^{l}(\theta) \sqrt{1+\alpha r^{2}\left(\theta^{\prime}\right)^{2}}-\frac{1}{4} c^{2} \alpha r^{2 k+4}\left(\theta^{\prime}\right)^{2}+\ldots\right] .
$$

The first term in [.] gives rise to the equation of motion for a pure slipping mode $\theta_{0}(r)$ with no $u$ dependence discussed in section 4.1.2. If we make an ansatz that $\theta=\theta_{0}+\delta \theta$ with $\theta_{0} \sim r^{\Delta_{-}}$, the correction term in the equation of motion demands that $\delta \theta$ is of order 
$r^{2 k+2+\Delta_{-}}$. Of course, the global regularity condition in the interior for the coupled problem will affect the $r^{\Delta_{+}}$coefficient in $\theta$ as well, and this term appears earlier in the expansion than the $r^{2 k+2+\Delta_{-}}$term.

It is straightforward to integrate the equations of motion numerically, once the smoothness condition in the IR is properly implemented. Explicit examples and a full phase diagram of these configurations, in particular addressing the question whether the connected or the disconnected configuration has the smaller area, have been presented in [17]. One interesting new phenomenon that occurs in these examples is that for a certain range of local boundary data more than one connected regular minimal area exists, that is the global terms aren't unique but can be chosen from a discrete family.

\section{Acknowledgments}

We'd like to thank Michael Spillane for collaboration during initial stages of this work. We'd also like to thank Han-Chih Chang, Carlos Hoyos, Kristan Jensen, Rafe Mazzeo, and Andrew O'Bannon for useful discussions. The work of A. Karch was supported in part by U.S. DOE grant No. DE-FG02-96ER40956. The work of R. Graham was partially supported by NSF grant \# DMS 1308266.

Open Access. This article is distributed under the terms of the Creative Commons Attribution License (CC-BY 4.0), which permits any use, distribution and reproduction in any medium, provided the original author(s) and source are credited.

\section{References}

[1] J.M. Maldacena, The Large- $N$ limit of superconformal field theories and supergravity, Adv. Theor. Math. Phys. 2 (1998) 231 [Int. J. Theor. Phys. 38 (1999) 1113] [hep-th/9711200] [INSPIRE].

[2] S.S. Gubser, I.R. Klebanov and A.M. Polyakov, Gauge theory correlators from noncritical string theory, Phys. Lett. B 428 (1998) 105 [hep-th/9802109] [INSPIRE].

[3] E. Witten, Anti-de Sitter space and holography, Adv. Theor. Math. Phys. 2 (1998) 253 [hep-th/9802150] [INSPIRE].

[4] C. Fefferman and C. R. Graham, Conformal invariants, Astérisque, Numero Hors Serie (1985) 95.

[5] S. de Haro, S.N. Solodukhin and K. Skenderis, Holographic reconstruction of space-time and renormalization in the AdS/CFT correspondence, Commun. Math. Phys. 217 (2001) 595 [hep-th/0002230] [INSPIRE].

[6] C. Fefferman and C. R. Graham, The Ambient Metric, Princeton University Press, Princeton U.S.A. (2012).

[7] J.M. Maldacena, Wilson loops in large-N field theories, Phys. Rev. Lett. 80 (1998) 4859 [hep-th/9803002] [INSPIRE].

[8] S.-J. Rey and J.-T. Yee, Macroscopic strings as heavy quarks in large-N gauge theory and anti-de Sitter supergravity, Eur. Phys. J. C 22 (2001) 379 [hep-th/9803001] [INSPIRE]. 
[9] A. Karch and E. Katz, Adding flavor to AdS/CFT, JHEP 06 (2002) 043 [hep-th/0205236] [INSPIRE].

[10] S. Ryu and T. Takayanagi, Holographic derivation of entanglement entropy from AdS/CFT, Phys. Rev. Lett. 96 (2006) 181602 [hep-th/0603001] [INSPIRE].

[11] C.R. Graham and E. Witten, Conformal anomaly of submanifold observables in AdS/CFT correspondence, Nucl. Phys. B 546 (1999) 52 [hep-th/9901021] [INSPIRE].

[12] A. Karch, A. O'Bannon and K. Skenderis, Holographic renormalization of probe D-branes in AdS/CFT, JHEP 04 (2006) 015 [hep-th/0512125] [INSPIRE].

[13] H.B. Lawson Jr., Lectures on Minimal Submanifolds Vol. I, Publish or Perish, Inc., Berkeley U.S.A. (1980).

[14] A. Karch and L. Randall, Open and closed string interpretation of SUSY CFT's on branes with boundaries, JHEP 06 (2001) 063 [hep-th/0105132] [INSPIRE].

[15] K. Skenderis and M. Taylor, Branes in AdS and p p wave space-times, JHEP 06 (2002) 025 [hep-th/0204054] [INSPIRE].

[16] N. Evans and K.-Y. Kim, Vacuum alignment and phase structure of holographic bi-layers, Phys. Lett. B 728 (2014) 658 [arXiv:1311.0149] [INSPIRE].

[17] H.-C. Chang, Minimal submanifolds asymptotic to $A d S_{4} \times S^{2}$ in $A d S_{5} \times S^{5}$, JHEP 04 (2014) 037 [arXiv: 1310.5734] [INSPIRE]. 